\title{
MINDERMANN, Arend, Adel in der Stadt des Spätmittelalters. Göttingen und Stade 1300 bis 1600
}

Joseph Morsel

\section{OpenEdition}

\section{Journals}

Édition électronique

URL : http://journals.openedition.org/ifha/1298

DOI : $10.4000 /$ ifha. 1298

ISSN : 2198-8943

\section{Éditeur}

IFRA - Institut franco-allemand (sciences historiques et sociales)

\section{Référence électronique}

Joseph Morsel, « MINDERMANN, Arend, Adel in der Stadt des Spätmittelalters. Göttingen und Stade 1300 bis 1600 », Revue de l'IFHA [En ligne], Date de recension, mis en ligne le 01 janvier 2000, consulté le 22 septembre 2020. URL : http://journals.openedition.org/ifha/1298; DOI : https://doi.org/10.4000/ifha. 1298

Ce document a été généré automatiquement le 22 septembre 2020.

(C)IFHA 


\title{
MINDERMANN, Arend, Adel in der Stadt des Spätmittelalters. Göttingen und Stade 1300 bis 1600
}

\author{
Joseph Morsel
}

1 Les rapports entre la noblesse et la ville constituent un thème de recherche de plus en plus important outre-Rhin (BullMHFA, 32, 1996, p. 33-53), auquel A.M. apporte une intéressante contribution. L'objet de sa thèse de doctorat est la présence de nobles campagnards au sein de la ville, présence tant spatiale que matérielle et personnelle, à l'encontre de l'opposition qu'une historiographie ancienne mais encore résistante postulait. La démarche d'A.M. n'est pas sans rappeler celle qu'A. Ranft avait mise en œuvre dans son étude des " sociétés de noblesse » (BullMHFA, 32, 1996, p. 115-116) : examen successif de deux cas d'espèce (ici les villes de Göttingen et de Stade) à partir d'une grille identique (ici : " noblesse et seigneur urbain : les curiæ urbaines de la noblesse ", " noblesse et conseil de ville ", " noblesse et Église ", " tournois "; l'organisation interne de chacun de ces points est elle-même très répétitive d'une ville à l'autre, bien que les différences locales contraignent à de petites adaptations), puis comparaison entre elles et avec d'autres cas allemands (ici principalement Hanovre, mais pas seulement), toujours en suivant la même grille (ce que n'avait pas fait Ranft). Le choix des deux villes de base (Göttingen et Stade), quoique justifié par A.M. en raison de leurs ressemblances (villes hanséatiques, même ancienneté, présence de consules depuis la même époque, institutions municipales comparables, présence d'un château seigneurial, expulsion du seigneur au XIVe s.) et de leurs dissemblances (ville de l'intérieur au sein d'un espace topographiquement cloisonné vs. ville côtière ouverte sur l'extérieur), reste peu convaincant : rien de tout ceci ne suggère a priori que ces villes constituent un lieu d'examen significatif des rapports entre ville et noblesse ; il eût été sans doute plus pertinent de choisir (ou du moins de justifier le choix) des villes connues pour leurs options ouvertement favorables ou hostiles à la noblesse (ou une de chaque), ce qui pouvait faire des rapports ville/noblesse un enjeu social à l'époque étudiée, et non un questionnement quelque peu gratuit. 
2 De fait, la répétition du questionnement sur les trois parties du travail permet certes de s'y retrouver aisément, mais elle donne aussi à la démarche un caractère un peu schématique sinon artificiel : on peut sans problème parler de plan "à tiroirs ", que nous reprochons normalement à nos étudiants parce qu'il découle soit de l'absence de problématique - ce qui n'est pas vraiment le cas ici -, soit d'une projection sur le matériau d'une problématique ou de catégories élaborées hors-contexte (c'est-à-dire de manière an-historique). La présentation par A.M. de sa problématique est ainsi caractéristique : il s'agit avant tout de combler un "vide » historiographique, ou de construire le pendant de la « présence attestée des bourgeois - et ici en particulier des patriciens - dans ce que [Otto] Brunner appelle « le monde de la seigneurie rurale > » en montrant la présence des nobles dans le monde de la bourgeoisie urbaine. Nulle part n'apparaît d'interrogation sur la validité de l'emploi des catégories « ville », " noblesse ", « Église ", etc., ni sur l'hypothèse que ces catégories puissent se définir les unes par rapport aux autres, ni encore sur la possibilité heuristique d'étudier la noblesse dans les villes à travers les rapports entre des nobles individuels et des instances urbaines particulières (institutions municipales, établissements ecclésiastiques urbains, pratiques a priori considérées comme nobiliaires et accomplies dans les villes, etc.), ni enfin sur l'écart entre pratiques et discours. Bref, si l'objet est légitime (ville/noblesse), la problématique est par trop substantialiste pour pouvoir apporter à l'objet autre chose que du matériau de réponse qui attende un traitement adéquat.

3 Pour ce qui est du matériau, toutefois, l'ouvrage d'A.M. fournit un grand nombre de données tirées d'un volume conséquent de sources exploitées, publiées ou non. C'est ce qui fait l'intérêt majeur du travail. Le caractère probablement le plus important - ne serait-ce que parce qu'il était jusqu'alors le moins étudié - de la présence aristocratique en ville est la détention de portions notables du sol urbain, occupées par des " cours » (Höfe $=$ curiæ), tenues en fief castral et le plus souvent à proximité du château seigneurial et donc à la périphérie (ce que montrent clairement les cartes établies par A.M.) : la concentration spatiale est particulièrement nette à Göttingen. L'évolution de cette implantation urbaine est elle aussi significative : parcellisation, accensement, vente progressive, au XIVe s., principalement aux conseils de ville - mais aussi, par donation, aux ordres mendiants, principalement les Franciscains, ce qui a évidemment comme conséquence l'implantation périphérique de ceux-ci dans la ville, que l'on a généralement tendance à rattacher à un modèle classique de lien avec les portes et avec les couches populaires. Inversement, les conseils s'évertuent à empêcher ou rendre difficile l'acquisition de biens intra muros par les nobles, mais cette absence foncière ne signifie toutefois pas une absence personnelle : on observe en effet que des nobles font partie de la bourgeoisie (ils constituent même par moment jusqu'à $20 \%$ des nouveaux bourgeois), résidente ou pas (il s'agit alors de « bourgeois forains »). Il était en effet de l'intérêt des villes de s'assurer de la fidélité et de l'aide d'une partie de l'aristocratie afin de faire face à d'éventuelles agressions, dans le cadre de faides par exemple, et l'on rencontre ainsi des membres de la petite aristocratie au service soldé de la ville. En outre, les donations et fondations pieuses, avec la contrepartie de la commémoration, assuraient aussi, d'une certaine manière, une présence perpétuelle dans la ville, quoique de manière purement spirituelle. L'organisation de tournois en ville, enfin, pourrait être considérée comme une autre forme de présence aristocratique en ville, de manière festive/ostentatoire, mais les tournois organisés à Göttingen et Stade de la fin $\mathrm{du}$ XIIIe au XVIe s. sont peu significatifs de ce point de vue : ils sont peu nombreux (sept en tout), organisés essentiellement par le prince local (duc de Brunswick et archevêque 
de Brême) à des fins tactiques (assembler les nobles locaux autour du prince), et les citadins semblent les considérer essentiellement comme des formes de mise en valeur face aux autres villes, dont on invite des représentants : bref, deux logiques sociales parallèles et qui concernent surtout les rapports entre ville et princes locaux. Au total, par conséquent : des résultats à connaître, mais à creuser et à interpréter de manière plus problématisée.

Joseph MORSEL 